\title{
INDEX OF NATURAL OBJECTS
}

Achillea formosa (Boiss.) Sch. Bip. subsp. amanica (Rech. f.) Ehrend. \& Y.P. Guo, No. 2, 300

Aconitum karakolicum Rapaics, No. 2, 265

Albizzia julibrissin Durazz, No. 2, 268

Alhagi pseudalhagi (MB) Desv., No. 2, 268

Allium sativum L., No. 2, 270

Annona muricata L., No. 2, 321

Arachis hypogaea L., No. 2, 268

Aster himalaicus, No. 2, 309

Astragalus mucidus Bunge, No. 2, 250

Astragalus nuciferus L., No. 2, 268

Astragalus sieversianus Pall., No. 2, 268

Astragalus sp., No. 2, 268

Cassia angustifolia Vahl, No. 2, 268

Cedrus deodara (Roxb.) Loud., No. 2, 272

Centaurea scabiosa L., No. 2, 311

Cercis canadensis L., No. 2, 268

Cicer sativum L., No. 2, 268

Cinnamomum sreticulatum Hay, No. 2, 220

Cinnamomum subavenium Miq, No. 2, 215

Cinnamoum reticulatum Hayata, No. 2, 306

Cirsium japonicum DC., No. 2, 279

Clematis vitalba L., No. 2, 313

Colutea orbiculata Sumn., No. 2, 268

Colutea paulsenii Freyn., No. 2, 268

Corylopsis willmottiae Rehd. et Wils, No. 2, 194

Crotalaria alata L., No. 2, 268

Dendrobium thyrsiflorum Rchb., No. 2, 275

Ferula kuhistanica, No. 2, 182

Ferula tenuisecta, No. 2, 182

Genista tricuspidata Desf., No. 2, 277

Gleditsia macracantha, No. 2, 179

Glycine hispida (Mnch)., No. 2, 268

Gymnocladus dioca (L.) Koch, No. 2, 268

Halimodendron halodendron (Pall) Voss., No. 2, 268

Inula japonica Thunb., No. 2, 303
Juglans mandshurica Maxim., No. 2, 254

Linum culinaris Medik, No. 2, 268

Littorina sitkana, No. 2, 197

Maackia amurensis, No. 2, 197

Machilus odoratissima Nees, No. 2, 201

Mallotus apelta Muell. Arg., No. 2, 218

Medicago sativa L., No. 2, 268

Melodorum fruticosum Lour., No. 2, 292

Nelumbo nucifera Gaertn. cv. Rosa-plena, No. 2, 316

Nepeta suavis, No. 2, 234

Onobrynchis pulchella Schrenk., No. 2, 268

Penicillium sp. from Acanthus ilicifolius Linn., No. 2, 322

Phaseolus aureus Roxb., No. 2, 268

Phaseolus vulgaris L., No. 2, 268

Physalis ixocarpa Brot. (= Physalis philadelphica Lam.), No. 2, 295

Plantago depressa, No. 2, 165

Plantago lanceolata L., No. 2, 288

Plantago major L., No. 2, 288

Plantago media, No. 2, 165

Potentilla alba L., No. 2, 290

Salsola collina Pall., No. 2, 257

Scutellaria baicalensis Georgi, No. 2, 190

Senecio scandens Buch.-Ham., No. 2, 243

Serissa serissoides (DC.) Druce, No. 2, 176

Tagetes erecta, No. 2, 281

Tanacetum kotschyi (Boiss.) Grierson, No. 2, 297

Tanacetum vulgare L., No. 2, 284

Usnea sp., No. 2, 203

Verbena officinalis L., No. 2, 319

Vexibia alopecuroides L., No. 2, 268

Vexibia pachycarpa C.A., No. 2, 268

Vicia tetrasperma (L.) Moench, No. 2, 268

Euphorbia franchetii B. Fedtsch., No. 2, 286

Euphorbia canescens L., No. 2, 286 